\title{
Childhood sexual and physical abuse: age at exposure modulates impact on functional outcome in early psychosis patients
}

\author{
L. Alameda $^{1,2 *}$, C. Ferrari ${ }^{2}$, P. S. Baumann ${ }^{1,2}$, M. Gholam-Rezaee ${ }^{3}$, K. Q. Do ${ }^{1}$ and P. Conus ${ }^{2}$ \\ ${ }^{1}$ Unit for Research in Schizophrenia, Center for Psychiatric Neuroscience, Department of Psychiatry, Lausanne University Hospital (CHUV), \\ Lausanne, Switzerland \\ ${ }^{2}$ Service of General Psychiatry, Treatment and Early Intervention in Psychosis, Program (TIPP-Lausanne), Lausanne University Hospital (CHUV), \\ Lausanne, Switzerland \\ ${ }^{3}$ Department of Psychiatry, Center for Psychiatric Epidemiology and Psychopathology, Lausanne University Hospital (CHUV), Lausanne, \\ Switzerland
}

Background. Evidence suggests a relationship between exposure to trauma during childhood and functional impairments in psychotic patients. However, the impact of age at the time of exposure has been understudied in early psychosis (EP) patients.

\begin{abstract}
Method. Two hundred and twenty-five patients aged 18-35 years were assessed at baseline and after 2, 6, 18, 24, 30 and 36 months of treatment. Patients exposed to sexual and/or physical abuse (SPA) were classified according to age at the time of first exposure (Early SPA: before age 11 years; Late SPA: between ages 12 and 15 years) and then compared to patients who were not exposed to such trauma (Non-SPA). The functional level in the premorbid phase was measured with the Premorbid Adjustment Scale (PAS) and with the Global Assessment of Functioning (GAF) scale and the Social and Occupational Functioning Assessment Scale (SOFAS) during follow-up.

Results. There were $24.8 \%$ of patients with a documented history of SPA. Late SPA patients were more likely to be female $(p=0.010)$. Comparison with non-SPA patients revealed that: (1) both Early and Late SPA groups showed poorer premorbid social functioning during early adolescence, and (2) while patients with Early SPA had poorer functional level at follow-up with lower GAF $(p=0.025)$ and lower SOFAS $(p=0.048)$ scores, Late SPA patients did not.

Conclusion. Our results suggest a link between exposure to SPA and the later impairment of social functioning before the onset of the disease. EP patients exposed to SPA before age 12 may present long-lasting functional impairment, while patients exposed at a later age may improve in this regard and have a better functional outcome.
\end{abstract}

Received 13 November 2014; Revised 10 March 2015; Accepted 17 March 2015; First published online 24 April 2015

Key words: Childhood trauma, first-episode psychosis, prospective, timing.

\section{Introduction}

In the last 15 years, substantial research has shown a high prevalence of childhood trauma among patients suffering from psychosis (Read et al. 2005). It has been suggested that exposure to traumatic experiences may represent an important risk factor for the later development of psychoses (Bebbington et al. 2004; Janssen et al. 2004; Spauwen et al. 2006) and a recent meta-analysis (Varese et al. 2012) showed that exposure to trauma is associated with a 2.8 -fold increased risk of developing psychosis in adulthood.

\footnotetext{
* Address for correspondence: Dr L. Alameda, Department of Psychiatry, Lausanne University Hospital (CHUV), rue de Caroline 2, 1003 Lausanne, Switzerland.

(Email: Luis.alameda-fernandez@chuv.ch)
}

Studies performed among psychosis patients have additionally shown a correlation between exposure to childhood adversity and various illness characteristics, including level of functioning (Lysaker et al. 2007; Conus et al. 2010). Functional outcome is a broad term that covers activities of daily living, vocational activities, social relationships, and degree of independence; it is a key element of outcome in psychotic disorders which greatly impacts the social disability burden (Green et al. 2000; Cornblatt et al. 2012). Deficit in functional level is often detectable before the onset of the illness, present in its early stages and it often persists, remaining relatively poor despite resolution of acute psychosis (Menezes et al. 2006). Some studies have focused on the link between exposure to childhood trauma and the later impairment of functional level in psychosis patients. One study (Lysaker et al. 2001) in a sample of chronic patients suffering 
from schizophrenia showed that history of sexual abuse was associated with poorer social role performance. A subsequent study by the same author (Lysaker et al. 2004) showed that exposure to sexual abuse was associated with vocational deficits and poorer work performance over time. Conus et al. (2010) showed in a large epidemiological cohort of 658 early psychosis (EP) patients, that exposure to sexual and/or physical abuse (SPA) was associated with poorer premorbid functional levels. Finally, a recent study (Stain et al. 2013) in a sample of 223 first-episode psychosis patients showed that childhood trauma was also associated with poorer premorbid functioning, and that exposure to interpersonal trauma was correlated with poorer social functioning in adulthood. Most studies examining the association between childhood trauma and deficits in functional level were, however, conducted in chronic samples (Lysaker et al. 2001, 2004; Schenkel et al. 2005; Davidson et al. 2009), and when performed in EP samples (Conus et al. 2010; Stain et al. 2013), they are limited by their cross-sectional design. In addition, none of them addressed the potential mitigating effect of age at the time of trauma exposure on later functional impairment.

Age at the time of trauma exposure has been highlighted as a potential determinant of the consequences of childhood adversity (Keiley et al. 2001; Thornberry et al. 2001; van der Vegt et al. 2009). Some studies have suggested that it may modulate its impact and its influence on later development of psychosis. Fisher et al. (2010) have shown in a case-control study performed on $182 \mathrm{EP}$ patients compared to 246 control subjects, that when physical abuse by the mother started during childhood, the risk to later develop psychosis was twice higher than in subjects where similar abuse started during adolescence. Arseneault et al. (2011) showed in a representative birth cohort of 2232 subjects, that exposure to maltreatment by an adult before age 7 multiplied by 3.48 [95\% confidence interval (CI) 1.93-6.26] the likelihood of reporting psychotic symptoms by the age of 12 , while exposure between ages 7 and 12 increased this risk only by 2.44 (95\% CI 1.10-5.39). There is, however, no study exploring the relationship between age at the time of trauma and subsequent functional level.

Considering these elements we planned the current study in order to examine, in a sample of $225 \mathrm{EP}$ patients treated in a specialized programme, the potential differential impact on functional level of exposure to SPA according to age at the time of exposure. We hypothesized that traumatic experiences occurring at an earlier age would induce a more severe and longer lasting impairment of functional level than trauma occurring later.

\section{Method}

\section{Procedure}

TIPP (Treatment and early Intervention in Psychosis Programme), a specialized EP programme, was launched in 2004 at the Department of Psychiatry CHUV, in Lausanne, Switzerland (Conus \& Bonsack, 2004; Baumann et al. 2013). Entry criteria to the program are: (i) age between 18 and 35 years; (ii) residence in the catchment area (Lausanne and surroundings; population about 300000 ); (iii) meeting threshold criteria for psychosis, as defined by the 'Psychosis threshold' subscale of the Comprehensive Assessment of At Risk Mental States (CAARMS; Yung et al. 2005) scale. Patients are referred to other treatment programmes if they have been taking antipsychotic medication for more than a total of 6 months, have psychosis related to intoxication or organic brain disease, or have an intelligence quotient $<70$.

A specially designed questionnaire (the TIPP Initial Assessment Tool: TIAT) is completed for all patients enrolled in the programme by case managers who have up to 100 contacts with patients during the 3 years of treatment. It allows assessment of demographic characteristics, past medical history, exposure to life events as well as symptoms and functioning. It is completed on the basis of information gathered with patients and their family over the first few weeks of treatment and can be updated during follow-up if new information emerges. Follow-up assessments exploring various aspects of treatment and co-morbidities as well as evolution of psychopathology and functional level are conducted by a research psychologist and by case managers after 2, 6, 12, 18, 24, 30 and 36 months in treatment. In particular, substance abuse diagnosis was assessed at each time-point and rated on the basis of DSM-IV criteria (APA, 2000). The Research and Ethics Committee of the Faculty of Biology and Medicine of Lausanne University granted access to TIPP clinical data for research purposes. This is a prospective study based on the 240 patients who had been enrolled in the programme and who had been in treatment for 36 months at the time of the study.

\section{Diagnostic assessment}

Diagnosis is the result of an expert consensus and is based on the following elements: (1) diagnosis reported by treating psychiatrist in all medical documents and at the end of any hospitalization; (2) longitudinal assessment by clinical case managers over the 3 years of treatment. The consensus diagnosis procedure realized by a senior psychiatrist and the senior psychologist who is in charge of scale-based assessment 
over the treatment period. They both review the entire file once after 18 months and again after 36 months or at the end of treatment and conduct a diagnostic process based on DSM-IV criteria (APA, 1994) discussing any unclear issue with the clinical case manager. In this paper, only the final diagnosis, defined at the end of TIPP treatment period, is considered.

\section{Assessment of history of past trauma}

Clinicians at TIPP are trained to conduct an extensive assessment of patients, including evaluation of exposure to traumatic life events. Case managers meet patients frequently over the treatment period, which provides a framework for the establishment of a trusting relationship, where extensive knowledge of patients' history can be gathered. TIAT contains a table where exposure to traumatic life events can be recorded as follows: (1) type of traumatic life event, rated as present or absent (sexual abuse, physical abuse, adoption, migration, separation of parents, loss of parent, sibling or close relative, conflict with partner, emotional neglect, victimization by peers, emotional abuse and a category for 'other' types of trauma); (2) time of occurrence in relation to psychosis stage (during the premorbid phase, during the prodrome or after onset of psychosis); (3) age at the time of first exposure to each one of the trauma that occurred and (4) single or repeated exposure to each one of the trauma that occurred. Sexual abuse refers to sexual molestation and/or rape. Physical abuse refers to physical attack or assault or being repetitively beaten by parents, relatives or caregivers. Considering that the clinicians who assessed exposure to life events did not rate the subjective perception of severity of the different forms of stressful events, we focused our analyses on exposure to sexual and physical abuse, considering such events would undoubtedly be considered as highly traumatizing by anyone (Ucok \& Bikmaz, 2007; Conus et al. 2010; Lysaker et al. 2010). In addition, while sexual and physical abuse may have different impact on patients, we grouped both types of trauma together due to the limited size of our sample, and patients were coded as exposed to 'SPA' (sexual and/or physical abuse) if they had been exposed either to sexual or physical abuse or both. Age at the time of exposure was categorized as follows on the basis of previous literature and in accord with the various phases explored through the Premorbid Adjustment Scale (PAS; Cannon-Spoor et al. 1982; see below): (1) Early SPA refers to exposure between birth and age 11, according to conventions employed by Fisher et al. (2010), Thornberry et al. (2001) and Widom et al. (2008); (2) Late SPA refers to exposure between ages 12 and 15. Patients who were exposed to trauma after age 15 were excluded from this study, considering they may already have been in the prodromal phase of their first psychotic episode (as described in the Results section).

\section{Premorbid adjustment}

Premorbid functioning was assessed with the PAS (Cannon-Spoor et al. 1982) on the basis of information obtained via interviews with patients and their relatives over the first few months of treatment. The PAS is an interview-based rating schedule designed to retrospectively assess functioning across four developmental phases of life, and before onset of the illness: childhood (up to 11), early adolescence (12-15), late adolescence (16-18), and adulthood (age $\geqslant 19$ ). It contains also a general section concerning the period prior the appearance of first psychotic symptoms. It contains social functioning items (e.g. withdrawal, peer relationships and socio-sexual functioning) and academic functioning items (scholastic performance, adaptation to school) which are rated from childhood through to late adolescence (except socio-sexual functioning which is not rated during childhood). Ratings on PAS are scored on a 0-6 Likert scale, with 0 indicating normal adjustment and 6 indicating severe impairment. The PAS has an established predictive and concurrent validity (Brill et al. 2008) and is one of the most widely used measures of premorbid functioning in schizophrenia.

In accordance with previous EP studies (Ucok et al. 2011; Chang et al. 2013), childhood and early adolescence periods only were considered for the assessment of pre-morbid phase, in order to be sure to avoid the potential impact of early prodromal manifestations of the illness on functional level. PAS scores for both childhood and early adolescence phase were determined, as well as a global score based on both of them. In addition, social and academic subscores were determined, in keeping with MacBeth \& Gumley (2008), for each of the assessment phases mentioned above. These nine scores were obtained according to the method developed by Cannon-Spoor et al. (1982), and ranged from 0 to 1 , a higher score indicating higher degree of impairment and hence lower functioning level.

\section{Level of functioning during follow-up}

The level of functioning at baseline and during followup was also assessed with the Global Assessment of Functioning (GAF; APA, 1994) scale and the Social and Occupational Functioning Assessment Scale (SOFAS; APA, 1994). While the SOFAS only takes the social and occupational functioning into account, the GAF also includes the level of symptoms. The 
psychometric properties of these instruments have been attested (Jones et al. 1995; Jovanović et al. 2008). All patients were assessed on the basis of the GAF and SOFAS scales at each time point (baseline, after 2, 6, $12,18,24,30$ and 36 months). Instead of relying on a categorical definition of functional recovery (which is dependent on one or several cut-off scores), theses scale were taken as continuous variables when comparing the three groups (Non-SPA, Early SPA, Late SPA).

\section{Statistical analysis}

To compare demographic characteristics among the three groups (Non-SPA, Early SPA, Late SPA) the $\chi^{2}$ test and one-way analysis of variance (ANOVA) were used for categorical and continuous variable respectively. Multiple comparisons were performed using the Bonferroni adjustment.

To measure the differences between groups in the premorbid adjustment scores and the level of functioning at baseline, we used a multiple linear regression model introducing the all available baseline measurements of PAS, GAF and SOFAS scores as dependent variables, SPA grouping as the main factor where the Non-SPA group was considered as the reference group in all regression models. The model was adjusted for age, sex, socioeconomic status (SES; subdivided as high, intermediate and low according to Chandola \& Jenkinson, 2000) and history of abuse and/or dependence on substances prior to the age of 16. To compare the level of functioning between groups during the follow-up we conducted linear mixed effects (LME) models taking all the observations by the individual into account throughout the followup assessed (at baseline, 2, 6, 12, 18, 24, 30 and 36 months after entry in TIPP). We adjusted the model with age of entry into the study, sex, SES, medical treatment adherence, history of substance abuse or dependence prior to age 16 , and current severe substance use, which was considered as such when patients scored $\geqslant 3$ in the Case Manager Rating Scale (CMRS; Drake et al. 1990) at the respective time point.

\section{Results}

\section{Patient sample}

Of the first 240 patients consecutively admitted to TIPP between 2004 and 2010, 15 were excluded from the study for the following reasons: (i) age at exposure to trauma was not available $(n=2)$; (ii) first exposure to SPA occurred during their prodomal phase $(n=5)$, after psychosis onset $(n=4)$ or after age $15(n=4)$. Therefore, baseline data on 225 patients was analysed at baseline, and on 169 patients after 36 months of treatment. Out of the 56 patients that were not assessed at the end of the programme, 37 were due to loss in follow-up or to transfer to other services due to moving out of the catchment area; 19 due to missing data regarding the level of functioning at that assessment. No differences regarding sex, premorbid functioning, duration of untreated psychosis, exposure to SPA in different periods, level of functioning at baseline (measured with SOFAS and GAF), age at entry into the programme or SES were detected between patients that completed the assessment and those who did not. We found that patients with a diagnosis of schizophrenia were more likely to be present in the group that was assessed up to the end of the programme $(p=0.026)$.

\section{Occurrence of of SPA, demographic and premorbid characteristics}

The diagnostic breakdown of the sample of 225 patients was as follows: $132(58.7 \%)$ were diagnosed with schizophrenia; 27 (12.0\%) with schizophreniform or brief psychotic disorder; 20 (8.9\%) with schizoaffective disorder; 15 (6.7\%) with bipolar disorder; nine $(4.0 \%)$ with major depression with psychotic features and $22(9.8 \%)$ with other disorders (psychosis not otherwise specified, delusional disorder, schizotypal personality disorder, autism).

Among the 225 patients, 169 (75.2\%) had no history of exposure to SPA while $56(24.8 \%)$ did. Within the SPA group, 41 (18.2\%) had been exposed to SPA before age 11 (Early SPA) and $15(6.6 \%)$ between ages 12 and 15 (Late SPA). Twenty-seven (12\%) patients had been exposed to sexual abuse; 41 (17.7\%) to physical abuse and $12(5.3 \%)$ to sexual and physical abuse. Fifteen $(6.6 \%)$ patients had been exposed to sexual abuse only; $29(12.8 \%)$ to physical abuse only. No association was found between diagnostic distribution and SPA subgrouping. SPA patients were more likely to be female $(p=0.020)$. Other demographic and premorbid factors are presented in Table 1.

\section{Premorbid adjustment and level of functioning at baseline in relation to exposure to SPA during childhood and early adolescence}

Linear regression models comparing the nine scores of PAS and the scores of GAF and SOFAS at baseline are presented in Table 2. Compared to the Non-SPA group, the Early SPA group showed a poorer level of functioning on the PAS Total score $(p=0.002)$, the PAS Childhood score $(p=0.003)$, the PAS Childhood Social score $(p=0.002)$, the PAS Early Adolescence score $(p=0.003$,$) the PAS Early Adolescence Social$ score $(p=0.001)$ and the PAS Social Total score $(p=<0.001)$. Compared to the Non-SPA group, the Late SPA group showed poorer PAS Early Adolescence scores $(p=0.006)$ and a poorer PAS 
Table 1. Demographic and premorbid factors related to early or late exposure to sexual and/or physical abuse

\begin{tabular}{|c|c|c|c|c|c|c|c|}
\hline & Statistic & $\begin{array}{l}\text { Total } \\
(n=225)\end{array}$ & $\begin{array}{l}\text { Non-SPA } \\
(n=169)\end{array}$ & $\begin{array}{l}\text { Early SPA } \\
(n=41)\end{array}$ & $\begin{array}{l}\text { Late SPA } \\
(n=15)\end{array}$ & Stat. (df) & $p$ value \\
\hline Age, years & Mean (S.E.) & $23.94(0.32)$ & $23.63(0.36)$ & $24.76(0.7)$ & $25.07(1.48)$ & $F(2,219)=1.4$ & 0.249 \\
\hline Sex, male & $\%(n)$ & $70.27(156)$ & $74.7(124)$ & $63.41(26)$ & $40(6)$ & $\chi^{2}(2)=9.06$ & 0.010 \\
\hline Years in school ( $\geqslant 9$ years) & $\%(n)$ & $80.61(158)$ & $82.19(120)$ & $78.95(30)$ & $66.67(8)$ & $\chi^{2}(2)=1.79$ & 0.407 \\
\hline History of suicide attempts & $\%(n)$ & $14.76(31)$ & $12.1(19)$ & $23.08(9)$ & $21.43(3)$ & $\chi^{2}(2)=3.52$ & 0.172 \\
\hline SUD & $\%(n)$ & $33.78(76)$ & $31.95(54)$ & $41.46(17)$ & $33.33(5)$ & $\chi^{2}(2)=1.34$ & 0.512 \\
\hline DUP, weeks & Mean (S.E.) & $46.25(8.27)$ & $33.81(7.99)$ & $79.48(27.22)$ & $37.46(16.64)$ & $F(2)=2.49$ & 0.086 \\
\hline
\end{tabular}

Non-SPA, Non-exposure to sexual and/or physical abuse; Early SPA, exposure to SPA in childhood, from birth to age 11 years; Late SPA, exposure to SPA during early adolescence, from ages 12-15; SUD, current substance abuse or dependence; DUP, duration of untreated psychosis; S.E., standard error.

Table 2. Regression analyses comparing the premorbid adjustment and the level of functioning at baseline between patients exposed to sexual and/or physical abuse early or late

\begin{tabular}{|c|c|c|c|c|c|c|c|c|}
\hline & \multicolumn{4}{|c|}{ Early SPA } & \multicolumn{4}{|c|}{ Late SPA } \\
\hline & $B$ & S.E. & $t$ & $p$ value & $B$ & S.E. & $t$ & $p$ value \\
\hline \multicolumn{9}{|l|}{ PAS scores } \\
\hline Total & 0.09 & 0.02 & 3.07 & 0.002 & 0.08 & 0.04 & 1.87 & 0.061 \\
\hline Childhood & 0.1 & 0.05 & 2.92 & 0.003 & 0.02 & 0.05 & 0.56 & 0.574 \\
\hline Childhood social & 0.1 & 0.03 & 3.02 & 0.002 & 0.07 & 0.05 & 1.45 & 0.148 \\
\hline Childhood academic & 0.02 & 0.04 & 0.53 & 0.593 & -0.04 & 0.06 & -0.67 & 0.497 \\
\hline Early adolescence & 0.09 & 0.03 & 2.96 & 0.003 & 0.13 & 0.04 & 2.76 & 0.006 \\
\hline Early adolescence: social & 0.12 & 0.03 & 3.20 & 0.001 & 0.15 & 0.05 & 2.54 & 0.011 \\
\hline Early adolescence: academic & 0.02 & 0.03 & 0.55 & 0.578 & 0.06 & 0.06 & 1.07 & 0.285 \\
\hline Social total & 0.12 & 0.03 & 3.47 & $<0.001$ & 0.12 & 0.05 & 2.27 & 0.024 \\
\hline Academic total & 0.03 & 0.03 & 0.97 & 0.332 & 0.00 & 0.05 & 0.14 & 0.888 \\
\hline \multicolumn{9}{|l|}{ Baseline scores } \\
\hline GAF & -3.67 & 2.93 & -1.25 & 0.212 & 4.73 & 4.31 & 1.09 & 0.273 \\
\hline SOFAS & -4.84 & 2.91 & -1.66 & 0.097 & 3.06 & 4.41 & 0.69 & 0.488 \\
\hline
\end{tabular}

PAS, Premorbid Adjustment Scale; GAF, Global Assessment of Functioning Scale; SOFAS, Social and Occupational Assessment of functioning Scale.

Abbreviations of SPA groups are given in Table 1 note. Model is adjusted for age, sex, socioeconomic status, premorbid substance abuse and/or dependence. Non-exposed group has been taken as group of reference.

Early Adolescence Social score $(p=0.011)$. There were no significant associations between Early or Late exposure to SPA and the academic domain of PAS. Premorbid substance abuse or dependence were significantly associated with a poorer PAS early adolescence academic score $(p=0.022)$. No significant differences were found regarding GAF and SOFAS scores at entry to the Tipp programme between Early SPA and Late SPA compared to Non-SPA patients.

\section{Relationship between exposure to SPA and functional outcome during follow-up}

Detailed results of LME models comparing the level of functioning at follow-up between Early, Late and
Non-SPA are given in Table 3. We observed a significant difference between Early SPA and Non-SPA groups regarding GAF $(p=0.025)$ and SOFAS $(p=$ 0.048 ) scores over all time points. There was, however, no significant difference in this regard between Late SPA and Non-SPA patients (see Fig. $1 a, b$ ).

\section{Discussion}

To our knowledge, this is the first study exploring the relationship between age at the time of exposure to SPA in childhood and functional level in a sample of EP patients followed-up prospectively. Two main findings stem from our results. First, age at the time of exposure to trauma modulates its impact on later 
Table 3. Linear mixed effects model comparing the level of functioning after 2, 6, 12, 18, 24, 30 and 36 months of treatment between patients exposed to sexual and/or physical abuse early or late

\begin{tabular}{|c|c|c|c|c|c|c|c|c|}
\hline & \multicolumn{4}{|l|}{ GAF } & \multicolumn{4}{|c|}{ SOFAS } \\
\hline & $B$ & S.E. & $t$ & $p$ value & $B$ & S.E. & $t$ & $p$ value \\
\hline Early SPA & -4.75 & 2.10 & -2.25 & 0.025 & -4.13 & 2.08 & -1.98 & 0.048 \\
\hline Late SPA & 0.62 & 3.49 & 0.17 & 0.859 & 1.53 & 3.46 & 0.44 & 0.658 \\
\hline
\end{tabular}

GAF, Global Assessment of Functioning Scale; SOFAS, Social and Occupational Assessment of functioning Scale.

Abbreviations of SPA groups are given in Table 1 note. Model adjusted for age, sex, socioeconomic status, lifetime substance abuse and/or dependence, current substance use and current treatment adherence.
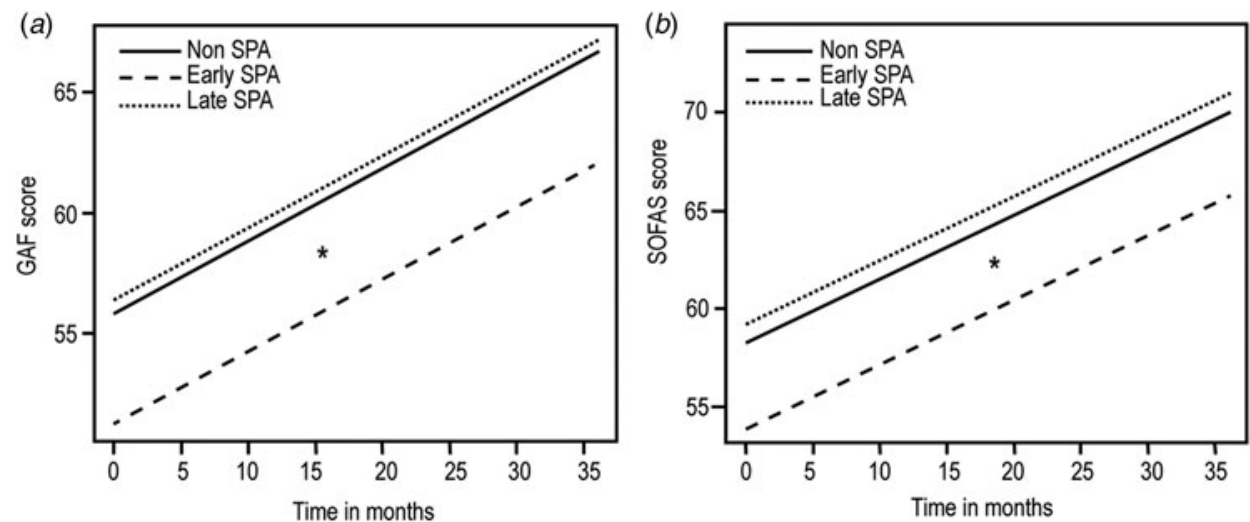

Fig. 1. Level of functioning measured with (a) the Global Assessment of Functioning Scale (GAF) and (b) the Social and Occupational Assessment of functioning Scale (SOFAS) with assessments at baseline, after 2, 6, 12, 18, 24, 30 and 36 months of treatment. Panels $(a)$ and $(b)$ represent a significant difference in GAF and SOFAS scores among Early SPA and Non-SPA groups; a parallel linear trend in time is fitted for all three groups to simplify the fitting process; by using a semi-parametric model we checked if this parallel trend in time is convincing for all groups, and the result supported our initial choice. Goodness of fit for fitted models are checked via graphical tools and were satisfactory for both models.

functional outcome. Second, impact of childhood trauma in the premorbid phase of psychotic disorders seems to be restricted to social domains while academic functioning remains similar to that of unexposed patients.

In addition to confirming previous findings that EP patients exposed to severe trauma in childhood have poorer functional outcome than non-exposed patients (Conus et al. 2010; Stain et al. 2013), our study suggests that age at the time of first exposure influences the long lasting effects of these deficits. In line with our hypothesis, we found thatcompared to non-exposed patients, those exposed to SPA before age 11 displayed a functional impairment that was maintained at follow-up, while patients exposed at a later age had improved over the treatment period. Indeed, Late SPA patients went from a functional level that was similar to that of Early SPA patients in the premorbid phase to a functional level that was similar to, if not better than, that of non-exposed EP patients throughout the 3 years of treatment.
Although often reported, the mechanism underlying this link between trauma and later impaired functioning remains unclear. Several hypotheses have been formulated, based on various theoretical concepts. First, some authors have suggested a mediating role for meta-cogniton and theory of mind (ToM; Frith \& Corcoran, 1996) in this process. Evidence in this field suggests that early adversity such as sexual abuse (Lysaker et al. 2011b) and early institutional deprivation (Colvert et al. 2008) leads to a decreased capacity to recognize one's own emotions and the emotions of others, a key aspect of social cognition that has been shown to be specifically linked to deficits in social functioning (Pinkham et al. 2003; Bora et al. 2006; Lysaker et al. 2011a). Deficits in the capacity to 'think about thinking' may limit abilities to form a coherent image of oneself as functioning within a social context and may lead in turn to incapacity to resolve the conflicts and misunderstandings arising in daily life (Lysaker et al. 2011a). Such an incapacity to fine-tune to others in 
complex situations may contribute to poorer social and vocational adjustment. However, while suggested in various publications, the mediating role of ToM deficits per se in the connection between childhood trauma and functional outcomes, and its independence from symptomatology and other confounding factors, still lacks an evidence base and must be explored further. Second, other elements, such as cognitive deficits (Addington \& Addington, 2000; Aas et al. 2011); positive symptoms (especially hallucinations; Ross et al. 1994), attachment difficulties (Berry et al. 2008; Read \& Gumley, 2008) or social anxiety (Freeman et al. 2008; Freeman \& Fowler, 2009), may also play an important mediating role in functional outcome. They have been shown to be related to childhood trauma and may well contribute to impairment in psychosocial functioning through increased tendency to isolation, deficit in communication skills or limited capacity to establish interpersonal relationships for example. Finally, hypotheses regarding the mechanisms leading to impaired functioning after trauma and the mediating effect of age the time of exposure in this phenomenon may also be formed at a neurobiological level. Recent research in our group on potential neurobiological mechanisms involved in the development of schizophrenia (Do et al. 2009; Steullet et al. 2014) suggests that environmental insults during development, through the induction of oxidative stress, may contribute to microcircuits and long pathway alterations which could be at the basis of various symptoms. In particular, oligodendrocytes (Monin et al. 2014) and parvalbumin interneurons (Cabungcal et al. 2013a, b), involved respectively in myelination and neural synchronization (Steullet et al. 2010), are particularly sensitive to early environmental insults. In an animal model with genetic deficit in glutathione, a major cellular antioxidant, the gene-environment interaction model revealed that additional stress in childhood and peripubertal ages, but not in adulthood, lead to severe and permanent parvalbumin interneuron alterations (Cabungcal et al. 2013a). Considering the dynamic and heterogeneous developmental trajectories of brain circuits, it can be hypothesized that early insults may differentially affect circuit connectivity compared to later insults, hence leading to different clinical phenotypes with distinct potential for recovery.

Whatever the mechanism may be, our results reveal a different trajectory for EP patients with childhood exposure to trauma depending on age at first exposure; in particular, they suggest that in the frame of a specialized programme, patients exposed at a later age manage to recover from the social functioning deficit they had, in the premorbid phase, compared to nonexposed EP patients. The comprehensive treatment offered in such settings combines medication and psychotherapy within the frame of the continuity of care warranted by the role of clinical case managers. This may be sufficient in patients who have had the chance to develop some basic relational tools before being exposed to trauma, but it seems ineffective in those where earlier occurrence of trauma has left them with very limited coping capacities. Until new findings open potential avenues for a differential approach at a neurobiological level, it seems therefore necessary to develop new psychotherapeutic strategies in order to help such patients face the challenges of social interactions.

Following the strategy proposed by MacBeth \& Gumley (2008), the separate analysis of the academic and social dimensions of the PAS revealed that there is a specific correlation between exposure to childhood trauma and the social but not the academic dimension of premorbid functioning. This is in contradiction with one study (Stain et al. 2013), that found an association between exposure to trauma and impairment in both academic and social dimensions of PAS. The fact that they did not adjust their analyses for confounding factors such as SES and premorbid substance abuse may explain part of this difference. Our findings support previous evidence emphasizing the validity of dividing premorbid adjustment into its subcomponents (Allen et al. 2001; Norman et al. 2005) and point to a certain degree of independence between these two domains. They also suggest that being exposed to SPA early in life induces profound alterations of social interactions in early adolescence, which in turn may lead to avoidance of social contacts and withdrawal, which may precede the onset of psychosis in vulnerable individuals (Murphy et al. 2013).

Rate of childhood exposure to SPA in our sample $(24.8 \%)$ was slightly lower that that reported by others (Conus et al. 2010). However, when including patients who were excluded from the study, the rate of exposure rose to $29.5 \%$ which is in keeping with previous studies (Ucok \& Bikmaz, 2007; Conus et al. 2010). Similar to other publications (Neria et al. 2002), we found that women were more likely to be exposed to childhood SPA than men; this was mainly due to rate of exposure after age 12, otherwise the rate of early exposure to trauma is similar in both genders. In addition, we did not find differences in functioning at baseline between exposed and non-exposed patients. This is line with previous results (Conus et al. 2010), and could be explained by the ceiling effect of psychotic symptoms at the moment of entry to a specialized EP programme. Finally, contrary to what has been shown by others (De Bellis, 2002; Gordon, 2002), there was no association in our sample between childhood exposure to SPA and both diagnosis of 
substance abuse and a past history of suicide attempt (Conus et al. 2010).

This study has various limitations. First, exposure to SPA was assessed retrospectively in the current study, which may be particularly problematic in patients suffering from psychosis (Howard, 1993). However, exposure to SPA was assessed in the frame of 3 years of a rather intensive treatment and on the basis of information obtained from patients and their families in the frame of a trusting therapeutic relationship (Conus et al. 2010); all these elements may minimize the impact of recall bias existing in other forms of selfreport and cross-sectional research interviews (Morgan \& Fisher, 2007). Second, although age at the time of first exposure to trauma and the repetitive nature of this exposure were recorded, there was no information about the duration of the period of maltreatment. We therefore were not in a position to verify previous findings that only childhood-onset abuse that persists through adolescence may have an impact on later outcomes (Thornberry et al. 2001). Third, some forms of childhood adversity such as emotional and physical neglect, which have been shown to influence the level of functioning of patients with psychosis (Schenkel et al. 2005; Davidson et al. 2009) were not recorded, and may have played a role in the observations we made. Finally, our results stem from comparisons between subgroups of EP patients; due to the impact of the psychotic illness itself at the functional level, we therefore cannot conclude on the impact of childhood SPA trauma on the functional level in general, but only on its possible consequences when present in the past history of EP patients. In addition, it should be mentioned that the design of this study does not provide any information regarding a potential causal link between exposure to trauma in childhood and the later development of psychosis.

\section{Conclusions}

Based on a unique 3-year prospective study in EP, our results highlighted an association between exposure to SPA in childhood and the later impairment of social functioning before the onset of the disease in patients with psychosis. In addition, they show that patients exposed to such experiences at an early age present longlasting social and vocational deficits, while those exposed at later age may have a better potential for recovery. More research is needed to uncover the mechanisms explaining these differences, potentially leading to the development of various specific treatment strategies. In the meantime, current psychological approaches to history of childhood SPA in EP patients may need to be adapted, according to age at the time of first exposure.

\section{Acknowledgements}

We thank the Case Managers from Tipp Program for collecting these data and Martin Preisig for his mentorship. We thank our financial support: Swiss National Science Foundation (no. 320030_122419 to P.C. and K.Q.D.); National Center of Competence in Research (NCCR) 'SYNAPSY - The Synaptic Bases of Mental Diseases' from the Swiss National Science Foundation (no. 51AU40_125759 to KQD); Alamaya Foundation and Avina Stiftung. Most of all, we express our gratitude to all the patients for their enduring participation.

\section{Declaration of Interest}

None.

\section{References}

Aas M, Dazzan P, Fisher HL, Morgan C, Morgan K, Reichenberg A, Zanelli J, Fearon P, Jones PB, Murray RM, Pariante CM (2011). Childhood trauma and cognitive function in first-episode affective and non-affective psychosis. Schizophrenia Research 129, 12-19.

Addington J, Addington D (2000). Neurocognitive and social functioning in schizophrenia: a 2.5 year follow-up study. Schizophrenia Research 44, 47-56.

Allen DN, Kelley ME, Miyatake RK, Gurklis Jr. JA, van Kammen DP (2001). Confirmation of a two-factor model of premorbid adjustment in males with schizophrenia. Schizophrenia Bulletin 27, 39-46.

APA (1994). Diagnostic and Statistical Manual of Mental Disorders, 4th edn. American Psychiatric Association: Washington, DC.

APA (2000). DSMIV-TR. American Psychiatric Association: Washington, DC.

Arseneault L, Cannon M, Fisher HL, Polanczyk G, Moffitt TE, Caspi A (2011). Childhood trauma and children's emerging psychotic symptoms: a genetically sensitive longitudinal cohort study. American Journal of Psychiatry 168, 65-72.

Baumann PS, Crespi S, Marion-Veyron R, Solida A, Thonney J, Favrod J, Bonsack C, Do KQ, Conus P (2013). Treatment and Early Intervention in Psychosis Program (TIPP-Lausanne): implementation of an early intervention programme for psychosis in Switzerland. Early Intervention Psychiatry 7, 322-328.

Bebbington PE, Bhugra D, Brugha T, Singleton N, Farrell M, Jenkins R, Lewis G, Meltzer H (2004). Psychosis, victimisation and childhood disadvantage: evidence from the second British National Survey of Psychiatric Morbidity. British Journal of Psychiatry 185, 220-226.

Berry K, Barrowclough C, Wearden A (2008). Attachment theory: a framework for understanding symptoms and interpersonal relationships in psychosis. Behaviour Research and Therapy 46, 1275-1282.

Bora E, Eryavuz A, Kayahan B, Sungu G, Veznedaroglu B (2006). Social functioning, theory of mind and 
neurocognition in outpatients with schizophrenia; mental state decoding may be a better predictor of social functioning than mental state reasoning. Psychiatry Research 145, 95-103.

Brill N, Reichenberg A, Weiser M, Rabinowitz J (2008). Validity of the premorbid adjustment scale. Schizophrenia Bulletin 34, 981-983.

Cabungcal JH, Steullet P, Kraftsik R, Cuenod M, Do KQ (2013a). Early-life insults impair parvalbumin interneurons via oxidative stress: reversal by $\mathrm{N}$-acetylcysteine. Biological Psychiatry 73, 574-582.

Cabungcal JH, Steullet $\mathrm{P}$, Morishita $\mathrm{H}$, Kraftsik R, Cuenod M, Hensch TK, Do KQ (2013b). Perineuronal nets protect fast-spiking interneurons against oxidative stress. Proceedings of the National Academy of Sciences of the United States of America 110, 9130-9135.

Cannon-Spoor HE, Potkin SG, Wyatt RJ (1982). Measurement of premorbid adjustment in chronic schizophrenia. Schizophrenia Bulletin 8, 470-484.

Chandola T, Jenkinson C (2000). The new UK National Statistics Socio-Economic Classification (NS-SEC); investigating social class differences in self-reported health status. Journal of Public Health Medicine 22, 182-190.

Chang WC, Yee Man Tang J, Lai Ming Hui C, Hoi Yan Wong G, Kit Wa Chan S, Ho Ming Lee E, Yu Hai Chen E (2013). The relationship of early premorbid adjustment with negative symptoms and cognitive functions in first-episode schizophrenia: a prospective three-year follow-up study. Psychiatry Research 3, 353-360.

Colvert E, Rutter M, Kreppner J, Beckett C, Castle J, Groothues C, Hawkins A, Stevens S, Sonuga-Barke EJ (2008). Do theory of mind and executive function deficits underlie the adverse outcomes associated with profound early deprivation?: findings from the English and Romanian adoptees study. Journal of Abnormal Child Psychology 36, 1057-1068.

Conus P, Bonsack C (2004). Early intervention for the initial phase of psychotic disorders in Lausanne: what problems and what solutions? Revue Médicale de la Suisse Romande 124, 221-224.

Conus P, Cotton S, Schimmelmann BG, McGorry PD, Lambert M (2010). Pretreatment and outcome correlates of sexual and physical trauma in an epidemiological cohort of first-episode psychosis patients. Schizophrenia Bulletin 36, 1105-1114.

Cornblatt BA, Carrion RE, Addington J, Seidman L, Walker EF, Cannon TD, Cadenhead KS, McGlashan TH, Perkins DO, Tsuang MT, Woods SW, Heinssen R, Lencz T (2012). Risk factors for psychosis: impaired social and role functioning. Schizophrenia Bulletin 38, 1247-1257.

Davidson G, Shannon C, Mulholland C, Campbell J (2009). A longitudinal study of the effects of childhood trauma on symptoms and functioning of people with severe mental health problems. Journal of Trauma Dissociation 10, 57-68.

De Bellis MD (2002). Developmental traumatology: a contributory mechanism for alcohol and substance use disorders. Psychoneuroendocrinology 27, 155-170.

Do KQ, Cabungcal JH, Frank A, Steullet P, Cuenod M (2009). Redox dysregulation, neurodevelopment, and schizophrenia. Current Opinion in Neurobiology 19, 220-230.

Drake RE, Osher FC, Noordsy DL, Hurlbut SC, Teague GB, Beaudett MS (1990). Diagnosis of alcohol use disorders in schizophrenia. Schizophrenia Bulletin 16, 57-67.

Fisher HL, Jones PB, Fearon P, Craig TK, Dazzan P, Morgan K, Hutchinson G, Doody GA, McGuffin P, Leff J, Murray RM, Morgan C (2010). The varying impact of type, timing and frequency of exposure to childhood adversity on its association with adult psychotic disorder. Psychological Medicine 40, 1967-1978.

Freeman D, Fowler D (2009). Routes to psychotic symptoms: trauma, anxiety and psychosis-like experiences. Psychiatry Research 169, 107-112.

Freeman D, Gittins M, Pugh K, Antley A, Slater M, Dunn G (2008). What makes one person paranoid and another person anxious? The differential prediction of social anxiety and persecutory ideation in an experimental situation. Psychological Medicine 38, 1121-1132.

Frith CD, Corcoran R (1996). Exploring 'theory of mind' in people with schizophrenia. Psychological Medicine 26, 521-530.

Gordon HW (2002). Early environmental stress and biological vulnerability to drug abuse. Psychoneuroendocrinology 27, 115-126.

Green MF, Kern RS, Braff DL, Mintz J (2000). Neurocognitive deficits and functional outcome in schizophrenia: are we measuring the 'right stuff'? Schizophrenia Bulletin 26, 119-136.

Howard LM (1993). Allegations of abuse in psychotic patients. American Journal of Psychiatry 150, 839-840.

Janssen I, Krabbendam L, Bak M, Hanssen M, Vollebergh W, de Graaf R, van Os J (2004). Childhood abuse as a risk factor for psychotic experiences. Acta Psychiatrica Scandinavica 109, 38-45.

Jones SH, Thornicroft G, Coffey M, Dunn G (1995). A brief mental health outcome scale-reliability and validity of the Global Assessment of Functioning (GAF). British Journal of Psychiatry 166, 654-659.

Jovanović AA, GaSic MJ, Ivković M, Milovanović S, Damjanović A (2008). Reliability and validity of DSM-IV Axis $\mathrm{V}$ scales in a clinical sample of veterans with posttraumatic stress disorder. Psychiatria Danubina 20, 286-300.

Keiley MK, Howe TR, Dodge KA, Bates JE, Petti GS (2001). The timing of child physical maltreatment: a cross-domain growth analysis of impact on adolescent externalizing and internalizing problems. Development and Psychopathology 13, 891-912.

Lysaker PH, Buck KD, LaRocco VA (2007). Clinical and psychosocial significance of trauma history in the treatment of schizophrenia. Journal of Psychosocial Nursing and Mental Health Services 45, 44-51.

Lysaker PH, Erickson MA, Buck B, Buck KD, Olesek K, Grant ML, Salvatore G, Popolo R, Dimaggio G (2011a). Metacognition and social function in schizophrenia: associations over a period of five months. Cognitive Neuropsychiatry 16, 241-255.

Lysaker PH, Gumley A, Brune M, Vanheule S, Buck KD, Dimaggio G (2011b). Deficits in the ability to recognize one's own affects and those of others: associations with 
neurocognition, symptoms and sexual trauma among persons with schizophrenia spectrum disorders. Consciousness and Cognition 20, 1183-1192.

Lysaker PH, Meyer PS, Evans JD, Clements CA, Marks KA (2001). Childhood sexual trauma and psychosocial functioning in adults with schizophrenia. Psychiatric Services 52, 1485-1488.

Lysaker PH, Nees MA, Lancaster RS, Davis LW (2004). Vocational function among persons with schizophrenia with and without history of childhood sexual trauma. Journal of Traumatic Stress 17, 435-438.

Lysaker PH, Outcalt SD, Ringer JM (2010). Clinical and psychosocial significance of trauma history in schizophrenia spectrum disorders. Expert Review of Neurotherapeutics 10, 1143-1151.

MacBeth A, Gumley A (2008). Premorbid adjustment, symptom development and quality of life in first episode psychosis: a systematic review and critical reappraisal. Acta Psychiatrica Scandinavica 117, 85-99.

Menezes NM, Arenovich T, Zipursky RB (2006). A systematic review of longitudinal outcome studies of first-episode psychosis. Psychological Medicine 36, 1349-1362.

Monin A, Baumann PS, Griffa A, Xin L, Mekle R, Fournier M, Butticaz C, Klaey M, Cabungcal JH, Steullet P, Ferrari C, Cuenod M, Gruetter R, Thiran JP, Hagmann P, Conus P, Do KQ (2014). Glutathione deficit impairs myelin maturation: relevance for white matter integrity in schizophrenia patients. Molecular Psychiatry Published online: 26 August 2014. doi:10.1038/mp.2014.88.

Morgan C, Fisher H (2007). Environment and schizophrenia: environmental factors in schizophrenia: childhood trauma a critical review. Schizophrenia Bulletin 33, 3-10.

Murphy J, Shevlin M, Adamson G, Houston JE (2013). From sexual abuse to psychosis: a pilot study exploring the social deafferentation hypothesis and the mediating role of avoidance. Psychosis: Psychological, Social and Integrative Approaches 5, 36-47.

Neria Y, Bromet EJ, Sievers S, Lavelle J, Fochtmann LJ (2002). Trauma exposure and posttraumatic stress disorder in psychosis: findings from a first-admission cohort. Journal of Consulting and Clinical Psychology 70, 246-251.

Norman RM, Malla AK, Manchanda R, Townsend L (2005). Premorbid adjustment in first episode schizophrenia and schizoaffective disorders: a comparison of social and academic domains. Acta Psychiatrica Scandinavica 112, 30-39.

Pinkham AE, Penn DL, Perkins DO, Lieberman J (2003). Implications for the neural basis of social cognition for the study of schizophrenia. American Journal of Psychiatry 160, 815-824.

Read J, Gumley A (2008). Can attachment theory help explain the relationship between childhood adversity and psychosis? Attachment: New Directions in Psychotherapy and Relational Psychoanalysis 2, 1-35.

Read J, van Os J, Morrison AP, Ross CA (2005). Childhood trauma, psychosis and schizophrenia: a literature review with theoretical and clinical implications. Acta Psychiatrica Scandinavica 112, 330-350.
Ross CA, Anderson G, Clark P (1994). Childhood abuse and the positive symptoms of schizophrenia. Hospital and Community Psychiatry 45, 489-491.

Schenkel LS, Spaulding WD, DiLillo D, Silverstein SM (2005). Histories of childhood maltreatment in schizophrenia: relationships with premorbid functioning, symptomatology, and cognitive deficits. Schizophrenia Research 76, 273-286.

Spauwen J, Krabbendam L, Lieb R, Wittchen HU, van Os J (2006). Impact of psychological trauma on the development of psychotic symptoms: relationship with psychosis proneness. British Journal of Psychiatry 188, 527-533.

Stain HJ, Bronnick K, Hegelstad WT, Joa I, Johannessen JO, Langeveld J, Mawn L, Larsen TK (2013). Impact of interpersonal trauma on the social functioning of adults with first-episode psychosis. Schizophrenia Bulletin 40, 14911498.

Steullet P, Cabungcal JH, Kulak A, Kraftsik R, Chen Y, Dalton TP, Cuenod M, Do KQ (2010). Redox dysregulation affects the ventral but not dorsal hippocampus: impairment of parvalbumin neurons, gamma oscillations, and related behaviors. Journal of Neuroscience 30, 2547-2558.

Steullet P, Cabungcal JH, Monin A, Dwir D, O'Donnell P, Cuenod M, Do KQ (2014). Redox dysregulation, neuroinflammation, and NMDA receptor hypofunction: a 'central hub' in schizophrenia pathophysiology? Schizophrenia Research Published online: 4 July 2014. doi: http://dx.doi.org/10.1016/j.schres.2014.06.021.

Thornberry TP, Ireland TO, Smith CA (2001). The importance of timing: the varying impact of childhood and adolescent maltreatment on multiple problem outcomes. Development and Psychopathology 13, 957-979.

Ucok A, Bikmaz S (2007). The effects of childhood trauma in patients with first-episode schizophrenia. Acta Psychiatrica Scandinavica 116, 371-377.

Ucok A, Serbest S, Kandemir PE (2011). Remission after first-episode schizophrenia: results of a long-term follow-up. Psychiatry Research 189, 33-37.

van der Vegt EJ, van der Ende J, Ferdinand RF, Verhulst FC, Tiemeier H (2009). Early childhood adversities and trajectories of psychiatric problems in adoptees: evidence for long lasting effects. Journal of Abnormal Child Psychology 37, 239-249.

Varese F, Smeets F, Drukker M, Lieverse R, Lataster T, Viechtbauer W, Read J, van Os J, Bentall RP (2012). Childhood adversities increase the risk of psychosis: a meta-analysis of patient-control, prospective- and cross-sectional cohort studies. Schizophrenia Bulletin 38, 661-671.

Widom CS, Czaja SJ, Dutton MA (2008). Childhood victimization and lifetime revictimization. Child Abuse and Neglect 32, 785-796.

Yung AR, Yuen HP, McGorry PD, Phillips LJ, Kelly D, Dell'Olio M, Francey SM, Cosgrave EM, Killackey E, Stanford C, Godfrey K, Buckby J (2005). Mapping the onset of psychosis: the Comprehensive Assessment of At-Risk Mental States. Australian and New Zealand Journal of Psychiatry 39, 964-971. 